Anorganische Chemie

Prinzipien

von Struktur

und Reaktivität 



\section{James E. Huheey}

Anorganische Chemie

Prinzipien von Struktur und Reaktivität

übersetzt und bearbeitet von

Bertold Reuter

und

Brigitte Sarry

\begin{tabular}{|l|l}
\hline WE & Walter de Gruyter \\
$\mathrm{G}$ & Berlin $\cdot$ New York 1988
\end{tabular} 


\section{Titel der Originalausgabe}

Inorganic Chemistry

Third Edition

Principles of Structure

and Reactivity

James E. Huheey

University of Maryland

Copyright (C) 1983 by James E. Huheey

Harper \& Row, Publishers, Inc.

10 East 53 Street, New York, N.Y. 10022 USA

Übersetzer der deutschsprachigen Ausgabe

Prof. Dr.-Ing. Bertold Reuter

Prof. Dr. phil Brigitte Sarry

Institut für Anorganische und

Analytische Chemie der TU Berlin

Straße des 17. Juni 135

1000 Berlin 12

\section{CIP-Kurztitelaufnahme der Deutschen Bibliothek}

Huheey, James E.:

Anorganische Chemie : Prinzipien von Struktur u. Reaktivität /

James E. Huheey. Übers. u. bearb. von Bertold Reuter u.

Brigitte Sarry. - Berlin ; New York : de Gruyter, 1988

Einheitssacht.: Inorganic chemistry $\langle\mathrm{dt}$. $\rangle$

ISBN 3-11-008163-6

NE: Reuter, Bertold [Bearb.]

Copyright (C) 1988 by Walter de Gruyter \& Co., Berlin 30. Alle Rechte, insbesondere das Recht der Vervielfältigung und Verbreitung sowie der Übersetzung, vorbehalten. Kein Teil des Werkes darf in irgendeiner Form (durch Photokopie, Mikrofilm oder ein anderes Verfahren) ohne schriftliche Genehmigung des Verlages reproduziert oder unter Verwendung elektronischer Systeme verarbeitet, vervielfältigt oder verbreitet werden. Printed in Germany.

Satz und Druck: Tutte Druckerei GmbH, Salzweg-Passau.

Bindearbeiten: Lüderitz \& Bauer GmbH, Berlin.

Einbandentwurf: Hansbernd Lindemann, Berlin. 